Les facultés de médecine francophones subsahariennes constituent un réseau majeur dans le cadre du dispositif de formation des professionnels de santé dans cette région d'Afrique. Elles constituent également l'une des composantes essentielles des ressources de la communauté francophone en éducation des sciences de la santé, qui sont fédérées au sein de la Société internationale francophone d'éducation médicale (SIFEM). Dans ce contexte, le comité de rédaction de la revue Pédagogie Médicale et le conseil d'administration de la SIFEM sont heureux de pouvoir publier une traduction en français d'une importante étude internationale consacrée aux institutions de formation médicale en Afrique subsaharienne, ainsi que de l'éditorial d'accompagnement, dont les versions princeps ont récemment été publiées en anglais dans The Lancet. Ils remercient les éditions Elsevier d'avoir autorisé ces traductions et leur publication dans la revue.

La rédaction

\title{
Les soins de santé : une solution africaine
}

\section{Health care: an African solution*}

En 2006, le rapport mondial de la santé de l'Organisation mondiale de la santé (OMS) décrivait en détail l'état critique du système de soins de santé en Afrique subsaharienne. Les statistiques sont bien connues de quiconque s'intéressant à la santé à l'échelle mondiale. L'Afrique ne comptait que 2,3 prestataires de soins de santé pour 1000 personnes, chiffre à comparer à celui de l'Europe $(18,9$ pour 1000). En outre, en Afrique cet effectif devait faire face à $24 \%$ du fardeau mondial des maladies, ne bénéficiant que d'un pour cent des fonds mondiaux totaux destinés à la santé. La publication de ces statistiques a suscité de nombreuses autres publications ultérieures portant sur la santé en Afrique mais, jusqu'à présent, les solutions pratiques ont été rares.

La formation et la fidélisation du personnel médical, incluant les médecins, constituent la principale difficulté. La Commission du Lancet, Health professionals for a new century (Professionnels de la santé pour un siècle nouveau), a indiqué que c'était en fait un problème mondial. Le présent numéro du journal propose la traduction d'un article de politique de santé qui rapporte les résultats de l'étude sur les écoles de médecine en Afrique subsaharienne, dont la version princeps en anglais a été initialement publiée dans The Lancet ${ }^{[1]}$ et qui révèle les obstacles inhérents à l'éducation des étudiants en médecine en Afrique subsaharienne. L'enquête approfondie, menée par Fitzhugh Mullan et ses collègues auprès de 150 écoles de médecine, décrit des problèmes en matière d'accréditation, d'études supérieures et de coordination entre les ministères de la santé et de l'éducation; toutes ces lacunes contribuent à une dotation insuffisante de médecins, surtout dans les régions rurales. Le manque de professeurs et d'infrastructures et la question de la fidélisation des diplômés sont les points les plus inquiétants. Cet article est préoccupant mais il y a une lueur d'espoir.

Le 7 mars 2011, 240 personnes de 30 écoles de médecine d'Afrique et de 20 institutions des ÉtatsUnis se sont réunies à Johannesburg pour le premier symposium annuel de la Medical Education Partnership Initiative (MEPI - Initiative de partenariat pour l'éducation médicale). Jamais autant de parties prenantes ne s'étaient rassemblées dans le but d'améliorer l'éducation médicale en Afrique. La MEPI, dont la plupart des fonds proviennent d'une nouvelle initiative du Plan d'urgence présidentielle pour l'aide au SIDA (PEPFAR), vise à transformer l'éducation médicale en Afrique subsaharienne, en octroyant en 
premier lieu 130 millions de dollars répartis entre les écoles médicales de la région. Il est à signaler que toutes les allocations sont versées directement aux établissements africains, s'alignant ainsi sur le principe de l'administration du Président Obama, préconisant l'appropriation des projets par les pays bénéficiaires. Onze bourses programmatiques portant sur les cinq ans d'études médicales, à hauteur de deux millions de dollars US par an, et huit bourses financées par l' «US National Institute of Health» (Institut national de la santé des États-Unis) ont été octroyées. Cette initiative sera mise en œuvre par le biais de partenariats entre les bénéficiaires africains et d'autres écoles de médecine en Afrique ou aux États-Unis.

Le développement de l'effectif est la pierre angulaire de la MEPI et la fidélisation des diplômés est un objectif essentiel du programme. L'émigration des diplômés en médecine africains a largement contribué à la pénurie de médecins sur le continent; en 2005 , l'Organisation internationale pour les migrations estimait que, au terme des cinq années suivant l'obtention de son diplôme, un médecin africain sur cinq avait émigré dans un pays à revenu élevé. Pour encourager davantage de diplômés à rester sur place où ils sont indispensables, les écoles de la MEPI emploieront une combinaison de facteurs incluant l'éducation axée sur la communauté, une expérience précoce à l'exercice de la médecine en milieu rural, la création de filières professionnelles bien définies et un appui à des projets de recherche pertinents par rapport au contexte de la région. Les objectifs de la MEPI cherchant à améliorer l'infrastructure et à augmenter le corps professoral devraient accroître le nombre et la qualité des diplômés.

Le système des soins de santé en Afrique a également bénéficié d'une aide précieuse peu après le symposium de la MEPI, grâce à la réunion inaugurale de l'African Society for Laboratory Medicine (ASLM Société africaine des laboratoires médicaux) à Addis Abeba, Éthiopie. Financée par un fonds de 4,2 millions de dollars US octroyé par la PEPFAR, l'ASLM a pour but de coordonner des services de laboratoire de qualité supérieure à l'échelle du continent.

Tout comme les écoles de médecine, les services de laboratoire en Afrique manquent d'infrastructures et ne bénéficient pas d'une accréditation valable. Il n'existe pas non plus de corps professionnel de personnel qualifié. Mais à la fin de leur réunion, les délégués de l'ASLM espéraient avoir préparé la voie à une revitalisation des services de laboratoire. Les plans visant à professionnaliser les services, former le personnel et adopter une approche progressive et pragmatique pour accréditer les laboratoires devraient aboutir à un net progrès au regard des prestations de ces services. Il est permis d'espérer que cette approche interrompra l'engrenage sans fin associant les mauvais services de laboratoires au manque d'utilisation et d'investissement. La création de l'African Journal of Laboratory Medicine (Journal africain des laboratoires médicaux), la promotion de la recherche et le développement de réseaux permettant de partager et mettre à disposition des compétences techniques mondiales devraient aussi contribuer à soutenir et à fidéliser le personnel.

Bien que des difficultés en matière de logistique soient à anticiper à l'avenir pour la MEPI et l'ASLM, leur fondation mérite d'être reconnue dans le cadre d'efforts s'attachant à procurer des solutions africaines aux soins de santé au sein de l'Afrique. En fait, le mois de mars 2011 pourrait s'avérer être un point d'inflexion dans l'histoire du système de soins de santé en Afrique. Si tout va bien, ceux qui ont pris en charge ce changement pourront continuer à se laisser porter par l'enthousiasme, même si cela restera souvent un défi, et œuvrer ensemble pour un avenir africain dont la norme sera des soins de santé de qualité pour tous.

\section{The Lancet}

* La version princeps de cet éditorial a été publiée en anglais dans The Lancet (The Lancet. Health care: an African solution Lancet 2011; 377:1047). La présente traduction française a été effectuée par Odile Bosch 4602 Cornwallis Court 22309 Alexandria (VA), USA, et révisée au sein du comité de rédaction de Pédagogie Médicale; bien qu'elle ait été effectuée avec la permission des éditions Elsevier, elle n'a pas révisée sous l'égide de cet éditeur. 


\section{Références}

1. Mullan F, Frehywot S, Omaswa F, Buch E, Chen C, Greysen SR et al. Medical Schools in Sub-Saharan Africa. Lancet 2011;337:1113-21.

Pour la Commission de The Lancet voir: http:// www.lancet.com/education-of-health-professionals

Pour le Rapport mondial de la santé de l'OMS 2006 voir : http://www.who.int/whr/2006/whr06en.pdf (consulté en mars 2011).

Pour plus de renseignements sur la Medical Education Part-nership Initiative, voir : http://www.fic.nih.gov/ programs/training_grants/mepi/index.htm (consulté en mars 2011).

Pour 1' Organisation internationale pour les migrations, Rapport mondial sur les migrations 2005, voir : http:// publications.iom.int/bookstore/

index.php?main_page=product_info\&cPath $=37 \&$ produc ts_id=176

Pour plus de renseignements sur l'African Society for Laboratory Medicine, voir: http://www.afslm.org (consulté en mars 2011).

Pour l'article spécial sur les ressources humaines pour la santé de The Lancet, voir : Lancet 2008; 371:623-96. 